Musées, Patrimoine et Culture scientifiques et techniques

$114 \mid 2007$

novembre - décembre 2007

\title{
La médiation présencielle dans un musée des sciences
}

Florence Belaën et Marion Blet

\section{OpenEdition}

\section{Journals}

Édition électronique

URL : http://journals.openedition.org/ocim/704

DOI : 10.4000/ocim.704

ISSN : 2108-646X

Éditeur

OCIM

Édition imprimée

Date de publication : 1 novembre 2007

Pagination : 30-38

ISSN : 0994-1908

Référence électronique

Florence Belaën et Marion Blet, « La médiation présencielle dans un musée des sciences », La Lettre de I'OCIM [En ligne], 114 | 2007, mis en ligne le 03 février 2011, consulté le 30 avril 2019. URL : http:// journals.openedition.org/ocim/704; DOI : 10.4000/ocim.704 
et dans une démarche prospective consistant à lui attribuer éventuellement d'autres fonctions à l'avenir (4). Plusieurs questions ont motivé une réflexion générale : quelle est la particularité de l'offre de médiation humaine de type Cité des Sciences ? Comme il existe pour les expositions « un style Villette », existe-t-il un style de médiation «Villette »? Quelle est la plus value de cette offre pour les visiteurs ? Est-elle indispensable dans un musée des sciences du XXI ${ }^{\mathrm{e}}$ siècle ?

Pour répondre à ces questions, un travail d'analyse comparative entre différentes institutions internationales de culture scientifique (de taille de la CSI) a été mené (5) : malgré une diversité apparente et un langage propre à chaque institution, l'ensemble des activités relevant de la médiation culturelle se décline en une série de formats, que l'on retrouve de manière plus ou moins ostentatoire dans l'ensemble des institutions visitées. Trois grandes familles d'activités de médiation émergent à partir de trois types de relation médiateurs-visiteurs : nous y reviendrons à la fin de cet article. Le gigantisme de la Cité fait que l'ensemble de ces formats y est expérimenté et plus ou moins développé. Nous souhaitons partager ici ce travail de formalisation d'une activité dont le fonctionnement et les impacts demeurent difficiles à évaluer.

\section{Des difficultés à décrire la médiation présencielle}

Le monde des expositions bénéficie d'une réflexion approfondie sur les différents types de propositions muséographiques ; en effet des travaux comme ceux de Jean Davallon, Bernard Schiele ou encore Raymond Montpetit proposent des catégories d'expositions prenant en compte les modèles communicationnels portés par chacune d'entre elles. Même si ces catégories demeurent des modes de classement théoriques, elles permettent néanmoins de saisir une évolution des formes muséographiques, ainsi que l'émergence de genres dominants (exposition de collection, exposition thématique, exposition d'immersion...).

Les activités face-public restent une catégorie à part entière rattachée au monde des expositions et qui sont regroupées parfois sous le terme d'« activités culturelles », ou encore de " produits éducatifs » ou encore d'« animations » (6). Cette absence de langage commun pour décrire l'ensemble de ces propositions pourrait être interprétée comme un acte intentionnel de ne pas s'intéresser à "une offre faite de bouts de ficelle destinée à des groupes scolaires »... Mais l'expérience de la mise en œuvre de dispositifs d'évaluations spécifiques à cette offre a montré que les difficultés à décrire et formaliser ces offres sont réelles (Belaën, 2004) (7). Nous souhaiterions en évoquer ici quelques-unes.

Par définition, les activités qui relèvent de la médiation présencielle sont éphémères : elles ont lieu à un instant « $\mathrm{t}$ ». Quand l'activité est finie, aucune trace matérielle ne peut témoigner de ce qui s'est passé. Le dispositif de médiation directe est avant tout un dispositif « instable » : la qualité d'une action de médiation repose aussi bien sur le médiateur son aisance communicationnelle, son désir de transmettre, son degré de connaissance du sujet abordé, son expérience du public mais également son charisme, sa bonne humeur, son feeling avec les visiteurs, avec le sujet... - que sur la nature des visiteurs - leurs motivations, leurs attentes, le contexte de leurs venues... Ainsi chaque séance d'animation est unique et volatile...

Parce qu'elle relève d'une culture basée sur le principe de l'oralité et de l'expérience humaine, toute analyse dans le domaine se heurte à des difficultés d'appréciation tant pour le volet professionnel (descriptions des compétences, des savoir-faire et savoirêtre) qu'au niveau de l'évaluation de la réception (difficulté de verbalisation d'une expérience relevant d'une relation humaine...). Le fait que la médiation présencielle repose avant tout sur la rencontre entre un médiateur et des visiteurs nous conduit dans le domaine de la relation humaine et de la communication interpersonnelle. Si on ne souhaite pas appréhender la médiation directe, comme un seul dispositif de communication dont les objectifs se restreindraient à la transmission de connaissances, les études d'évaluation se heurtent à des difficultés

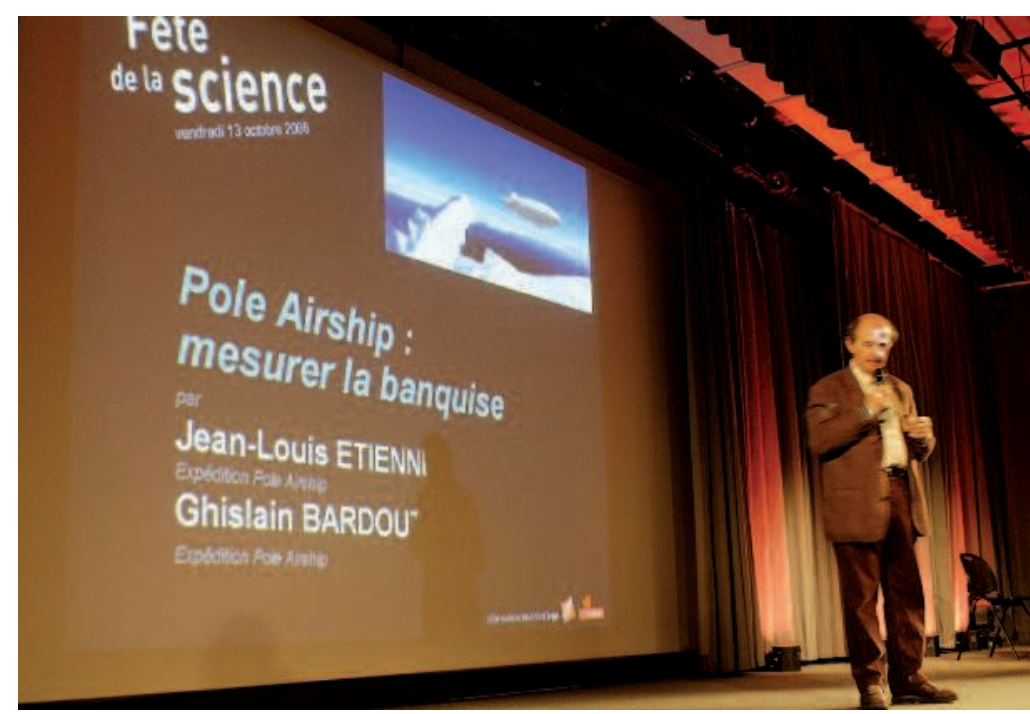

Certains chercheurs se révèlent être de très bons médiateurs.. (c) CSI/J.-P. Attal 
méthodologiques propres à ce type d'offre. Car si les visiteurs expriment volontiers un avis sur des objets matériels comme une exposition, un panneau, un site Internet en faisant remarquer qu' "elle est trop petite » ou «que le pannean est mal éclairé » ou «le site peu ergonomique», les remarques à l'issue d'une animation demeurent assez vagues et le plus souvent positives : "c'était bien ", "c'est sympa», accompagnées de nombreux encouragements pour le médiateur "il est dynamique », "il est très drôle », "il explique bien" ou encore "il a une voix très agréable »... Ces signes de sympathie et même parfois d'empathie du public vis-à-vis du médiateur expriment à la fois une forme de précaution, de gêne quant au fait de porter un jugement sur une personne qui est en situation de travail et une forme de remerciement vis-à-vis de quelqu'un qui a donné «d'une certaine façon » de son temps et qui s'est mis au niveau de ces interlocuteurs. Derrière ces formules de politesse se cachent également des difficultés que l'on peut ressentir à décrire une expérience qui relève avant tout d'une relation humaine.

Une autre difficulté repose sur le monde de référence de la médiation qui est au final multiple. Autant le monde de l'exposition repose sur une tradition ancrée datée de l'histoire du patrimoine (Schaer, 1993), autant la pratique de l'animation, au même titre que la culture scientifique et technique (Gaudillière, 2006), fait référence à plusieurs modèles communicationnels sans en privilégier un plus qu'un autre. Pour n'en citer que quelques-uns, les animations font bien sûr référence aux grandes démonstrations spectaculaires qui ont participé à la recherche scientifique : "venez voir tourner la terre sous vos pieds" comme l'annonçait Foucault au moment de son expérience au Panthéon en 1851. On retrouve l'esprit de ces expériences grandeur nature ouvertes aux publics avec le conservatoire national des Arts et Métiers. Cet esprit est toujours porté dans les exposés du Palais de la Découverte, qui rappellent la période des Expositions Universelles où des démonstrations mettaient en lumière les avancées techniques et scientifiques d'un pays (Eidelman, 1996). Une autre référence renvoie au mouvement de l'Éducation populaire. Démocratiser le savoir et la culture, rendre accessible au plus grand nombre, tel était le projet de démocratisation culturelle pour lequel l'animation socio-culturelle occupe une des premières places. Les dispositifs de médiation directe peuvent également rappeler inévitablement le monde de l'école où un médiateur est face à des apprenants, le premier se fixant pour objectif de transmettre du savoir, sans cadre imposé (pas de programme, pas de rapport d'autorité, pas d'évaluation en fin d'animation), avec juste quelques partis pris qui apparaissent pour le médiateur, être des incontournables à connaître.

Mais ces trois mondes de référence ne sont pas suffisants pour rendre compte de la pratique de médiation présencielle telle qu'elle a lieu aujourd'hui dans les musées ou centres de culture scientifique et technique. Faire une animation, c'est avant tout être sur une scène, sous les feux des projecteurs, que le dispositif technique soit conséquent ou pas. Les techniques d'animations sont aussi des techniques du spectacle et du théâtre. Savoir tenir son public en haleine, jouer de ses réactions, ouvrir et fermer la séance... La médiation présencielle fonctionne également avec les codes du théâtre (Raichvarg, 1993) et rappelle également les rituels des one man shows. Ainsi, parce que la médiation présencielle ne se limite pas à la seule transmission de connaissances, son fonctionnement demeure complexe : ce qui rend son analyse difficile à formaliser.

\section{Quelle est la valeur ajoutée de la médiation présencielle ?}

Malgré ces difficultés à rationaliser l'impact produit par la rencontre avec un médiateur, deux résultats d'études quantitatives ont souligné le bénéfice que peuvent en retirer certains visiteurs. L'Observatoire des Publics de la CSI a rapporté à plusieurs reprises que l'appréciation d'une visite, par une note attribuée par le visiteur, était plus élevée lorsque ce dernier avait assisté à une animation ${ }^{(8)}$. Dans un autre dispositif complémentaire d'évaluation quantitative

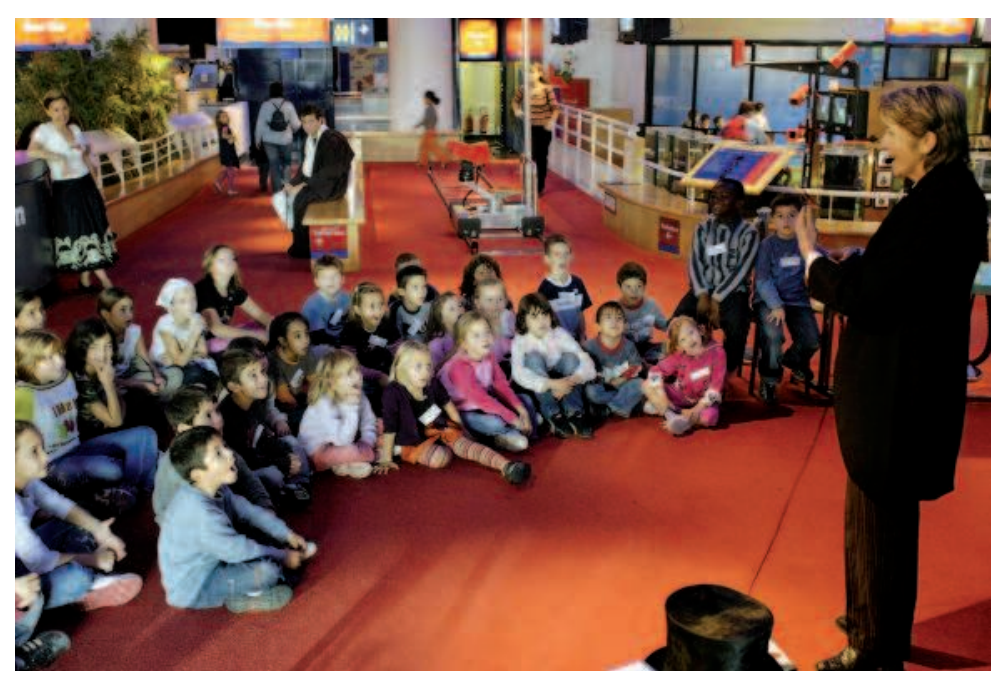

Les techniques théâtrales au service de la médiation () CSI/B. Baudin, Le Bar Floréal 
de cette offre, nous avons pu remarquer que les visiteurs qui choisissaient une animation, en complément de leur visite, fréquentaient souvent la Cité pour la $2^{\mathrm{e}}$ fois (Belaën, 2003). Une analyse des usages de ces dispositifs par les visiteurs a mis en lumière les atouts au regard du public de ce média reposant seulement sur "une voix et des gestes " (Wacquet, 2003) dans un monde dont la fonction même est de montrer des objets patrimoniaux ou techniquement sophistiqués. Sans prétendre à l'exhaustivité, nous souhaiterions aborder quelques-uns des effets que nous avons pu observer auprès de nos publics (Thievant, 1997, Belaën, 2003).

La médiation présencielle est avant tout un vecteur de compréhension efficace. On le sait, la mise en discours d'un phénomène, d'une théorie a été le premier processus de vulgarisation (Raichvarg, 1991). L'oral reste toujours le premier dispositif dans le domaine scolaire parce qu'il représente le moyen le plus bénéfique en termes de transmission de connaissances. Il est souvent plus simple et agréable que quelqu'un vous explique la théorie de l'Évolution ou de la Relativité générale que de devoir relire les auteurs. La médiation directe permet de proposer à voix haute une pensée, un raisonnement. En effet parce qu'elle repose seulement sur une parole et l'implication d'un corps, que tout se passe à l'instant « $\mathrm{t}$ ", en temps réel, l'animation ne fonctionne pas avec des effets de montages : sa force et sa puissance résultent donc de son emprise avec le réel ${ }^{(9)}$.

Le discours doit être limpide et compréhensible par l'auditoire dans sa plus grande diversité. Ainsi ces réalisations, construites selon des scénarios préalablement pensés, proposent à la fois du contenu et les liens logiques qui les animent. En effet, si la visite d'une exposition de manière autonome oblige le visiteur à retrouver la logique élaborée par le concepteur entre le cheminement spatial et conceptuel, le cadre d'une animation permet, quant à lui, au visiteur d'écouter une histoire et de se laisser porter dans cet univers imaginaire. La construction selon une narration portée à voix haute rend ces propositions structurées et structurantes pour les visiteurs. La médiation directe évite le zapping et invite au contraire à une communion autour d'un thème prédéfini.

Une autre force de ce média réside dans la possibilité de l'actualisation du discours. À la différence des produits finis comme les expositions, les films, les ouvrages dont la durée de conception ne peut se caler sur le rythme de l'actualité, les actions de médiation directe bénéficient d'une grande réactivité dans la

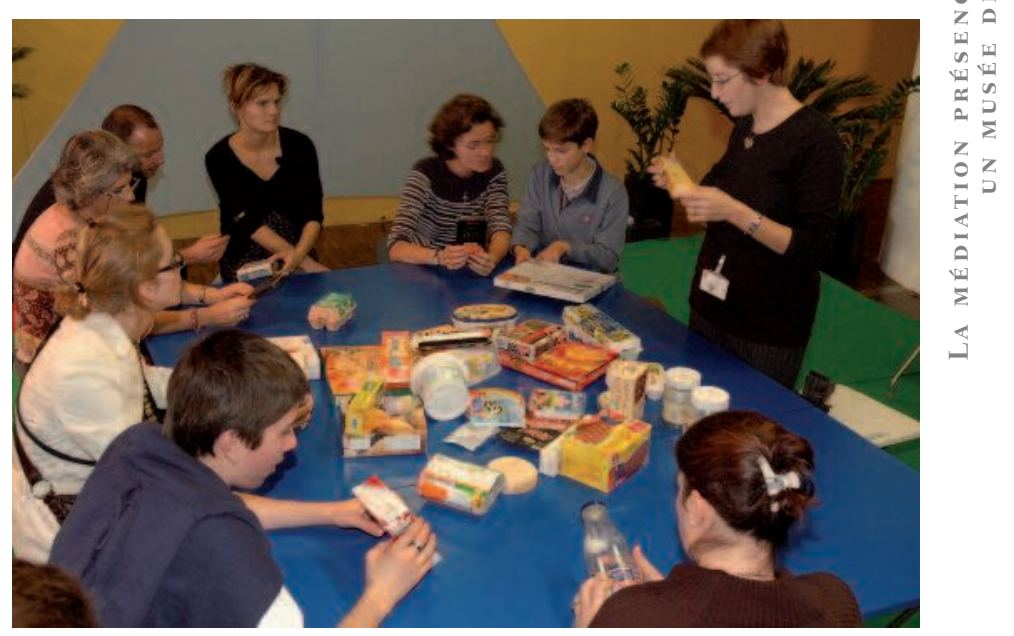

Les visiteurs sont de plus en plus acteurs dans les dispositifs de médiation. (c) CSI/M. Lamoureux

mise à jour des contenus. En effet le médiateur peut facilement rapporter la dernière découverte ou encore l'ultime controverse. Même si le temps de conception d'une animation est loin d'être négligeable du fait d'une grande maîtrise du sujet pour un rendu le plus simple qu'il soit, la réalisation pourrait s'apparenter à un traitement journalistique de l'information qui opère perpétuellement des ajustements en fonction de l'actualité scientifique. Comment ne pas évoquer le tsunami lorsqu'on présente un atelier sur la tectonique des plaques en ce début de l'année 2006 ? Comment ne pas parler de la grippe aviaire lorsqu'on réexplique les grandes épidémies ? Ce dispositif que l'on peut également qualifier de "flou » représente un autre avantage : celui de s'adapter in situ aux visiteurs. Répondre aux interrogations des visiteurs, réinvestir leurs expériences personnelles, prendre en considération leurs représentations préalables, que d'étapes indispensables dans tout processus d'apprentissage et de socialisation qui ne sont pas directement possibles dans les autres médias (télévision, exposition...).

Parce que les savoirs ne sont pas cloisonnés, la médiation directe permet également de prendre du recul par rapport à des sujets, de les replacer dans un contexte de production et de diffusion, de connaissances exponentielles. Les explications du médiateur, qui peut revenir à des fondamentaux (" une cellule, c'est quoi ? ) permet de relier une grande variété de données et d'informations, qui participent du bruit médiatique et que l'on ne parvient pas toujours à ordonner. 
Au-delà du traitement du sujet, l'impact de la médiation présencielle semble se distinguer des autres médias que l'on pourrait qualifier de « froids ». Un discours porté par une voix, par une personne présente laisse un fort souvenir : en effet le ton, le grain de la voix, la théâtralité, le rythme donnent vie au propos, marquent ainsi les esprits et laissent des traces dans la mémoire des visiteurs. L'écoute d'une parole identifiée et authentique a un fort impact, aussi parce que l'orateur dans son discours s'engage davantage dans ce qu'il dit : dans les conférences par exemple, il n'est pas rare qu'un chercheur au cours de son exposé porte un regard sur sa propre pratique, sur son métier. L'oral favorise davantage un discours personnalisé avec un parti pris que le discours du monde de l'écrit. Cette identification de la parole et cette prise de position sont appréciées des visiteurs, surexposés à des discours de tout genre, souvent très formatés par les contraintes techniques. Ces dispositifs favorisent le débat, la confrontation d'idées dans un cadre agréable où le visiteur n'a pas la crainte de se retrouver dans une situation d'échec. Enfin, un autre élément fort de ce média réside dans la socialisation du visiteur ${ }^{(10)}$. Plus que toute autre offre au musée, la médiation présencielle représente une véritable expérience humaine collective. Elle permet non seulement le dialogue avec un représentant de l'institution, mais également avec les autres visiteurs.

À l'heure où les musées s'engagent à procurer à leurs visiteurs de fortes expériences en pariant sur l'illusion technologique, les actions de médiation directe peuvent également proposer des expériences inoubliables : observer son propre ADN, faire l'expérience d'un fort voltage qui donne lieu à l'hérissement de ses cheveux sur la tête, fabriquer soi-même un instrument de musique... Parce que les dispositifs de médiation humaine demeurent des propositions qui tirent toute leur efficacité de la qualité de la rencontre qu'ils offrent aux visiteurs, ils permettent parfois à un groupe de visiteurs de s'enfermer dans un laboratoire pour y faire une expérience inédite. Ces expériences interactives laissent généralement des souvenirs très forts, elles peuvent d'ailleurs constituer une des sources de motivation à se déplacer jusque dans une institution culturelle...



Une relation du « 1 to 1 » entre le médiateur et le visiteur à la Cité de la Santé (๑) CSI/S. Chivet

\section{Des indicateurs à l'émergence de formats}

Notre travail de comparaison entre plusieurs institutions de culture scientifique et technique nous a conduit au constat suivant : les activités face-public même si leurs appellations peuvent varier ou que la place qu'elles occupent dans la programmation diffèrent selon les structures, fonctionnent selon des modes opératoires communs. En effet des critères sont apparus constitutifs des différentes formes d'activité de médiation présencielle. De ces différents indicateurs décrits dans le tableau ci-contre, une multitude de combinaisons est possible et donne lieu à des modes de communication, avec le public, extrêmement variés. Ainsi la médiation peut prendre diverses formes que nous appelons ici formats. Selon un point de vue synthétique, les formats de la médiation dépendent de l'objet de la médiation et de ses objectifs, du style adopté par le médiateur et de la position de celui-ci par rapport au public. 


\begin{tabular}{|c|c|c|}
\hline Indicateurs & Modalités de l'indicateur & Exemples de qualités \\
\hline Objet de la médiation & $\begin{array}{l}\text { Il est l'élément constitutif de la médiation. L'objet de } \\
\text { la médiation conditionne les paramètres du dispositif } \\
\text { (ou encore sa configuration). }\end{array}$ & $\begin{array}{l}\text { Objet } \\
\text { Discours } \\
\text { Expérience } \\
\text { Projection d'un film } \\
\text { Fond documentaire }\end{array}$ \\
\hline Fonction de la médiation & $\begin{array}{l}\text { Une activité de médiation peut répondre à } \\
\text { plusieurs fonctions en même temps. }\end{array}$ & $\begin{array}{l}\text { Accueillir/conseiller } \\
\text { Informer/expliquer } \\
\text { Montrer des objets } \\
\text { Faire participer, manipuler, } \\
\text { expérimenter, produire } \\
\text { Susciter l'échange, divertir }\end{array}$ \\
\hline Mode opératoire & Définit les conditions d'accès à l'offre de médiation. & $\begin{array}{l}\text { Gratuit } \\
\text { sur réservation } \\
\text { en groupe ou individuel } \\
\text { en continu }\end{array}$ \\
\hline Lieu de la médiation & $\begin{array}{l}\text { Il s'agit soit d'un lieu dédié à l'animation, } \\
\text { soit d'un lieu qui n'est pas spécifique pour celle-ci. }\end{array}$ & $\begin{array}{l}\text { Lieu fermé } \\
\text { Lieu semi-ouvert } \\
\text { Lieu polyvalent }\end{array}$ \\
\hline Durée de la médiation & $\begin{array}{l}\text { La temporalité de la médiation renvoie soit à un } \\
\text { produit fini ou au contraire une réponse personnalisée. }\end{array}$ & $\begin{array}{l}\text { Temps fini } \\
\text { Médiation personnalisée }\end{array}$ \\
\hline Les publics & $\begin{array}{l}\text { Cet indicateur concerne la nature du public } \\
\text { visé ainsi que le niveau. }\end{array}$ & $\begin{array}{l}\text { Publics individuels } \\
\text { Groupes scolaires } \\
\text { Publics spécifiques } \\
\text { Publics enfants } 3-5 \text { ans }\end{array}$ \\
\hline Outils de médiation & $\begin{array}{l}\text { Les outils viennent alimenter l'objet de médiation. } \\
\text { La nature de l'objet suppose donc des outils différents. }\end{array}$ & $\begin{array}{l}\text { Document(s) écrit(s) } \\
\text { Objet(s) } \\
\text { Éléments d'exposition } \\
\text { Ordinateurs et logiciels } \\
\text { Écran } \\
\text { Mise en scène }\end{array}$ \\
\hline Nature des médiateurs & $\begin{array}{l}\text { Ce critère renvoie à la formation du médiateur en } \\
\text { question ainsi qu'au nombre d'intervenants organisant } \\
\text { la rencontre avec le public. }\end{array}$ & $\begin{array}{l}\text { Professionnels de la médiation, } \\
\text { médiathécaires } \\
\text { Intervenant extérieur } \\
\text { représentant un domaine professionnel, } \\
\text { un domaine de recherche... }\end{array}$ \\
\hline Mode de médiation & $\begin{array}{l}\text { Postures médiateurs/visiteurs } \\
\text { Nombre de médiateurs }\end{array}$ & $\begin{array}{l}\text { Position frontale } \\
\text { Position médiane } \\
\text { Position en retrait }\end{array}$ \\
\hline Style de la médiation & Usages de langages formels & Humour, dramaturgie, narration \\
\hline Modèle de référence & $\begin{array}{l}\text { Les situations de médiation renvoient ou pas à } \\
\text { des modèles existants dans d'autres domaines. }\end{array}$ & $\begin{array}{l}\text { Situation pédagogique } \\
\text { Champ de la formation } \\
\text { Champ du spectacle } \\
\text { Entretien clinique... }\end{array}$ \\
\hline
\end{tabular}


L'objet de la médiation peut être un objet (un instrument scientifique, une œuvre d'art, un film, un fonds documentaire, un nouveau logiciel...), un discours (une théorie, une controverse...), une expérience (une expérience scientifique, une manipulation...). À partir de l'objet de médiation s'organise ensuite toute la configuration du dispositif (mode opératoire, lieu de la médiation, mode de références...). L'objet de la médiation est aussi inévitablement fortement relié à la fonction que l'on attend de cet acte de médiation : informer, expliquer, montrer des objets, manipuler, susciter l'échange...

L'ensemble du dispositif de médiation semble effectivement provenir de la nature de l'objet de la médiation. Malgré la diversité des différents indicateurs observés, trois formes de relation entre le médiateur et les visiteurs émergent. Chacune de ces formes de relations se caractérise par l'équilibre du couple médiateur/visiteurs, régi dans le dispositif par des lois de proxémie et de communication interpersonnelle.

L'interaction médiateur/visiteurs peut s'organiser de trois manières différentes à partir de la position du médiateur :
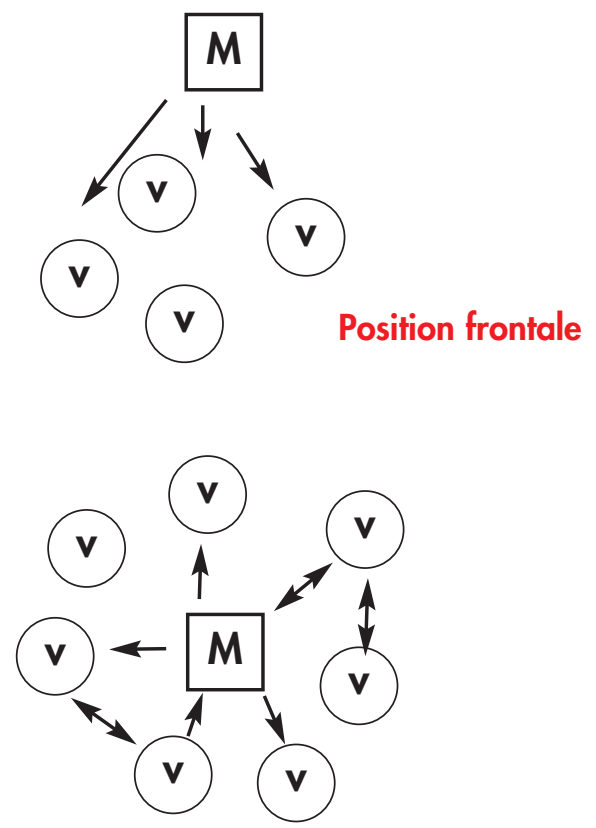

Position médiane

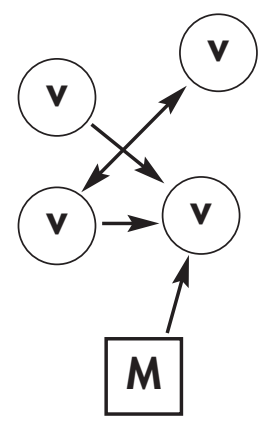

Position en retrait
Ainsi de l'organisation de ces différents indicateurs émergent des formats dominants qui nous apparaissent spécifiques dans le mode de communication proposé avec le public (voir tableau ci-contre).

Les grands formats de la médiation présencielle que l'on pourrait qualifier de traditionnels dans le monde de la culture scientifique et technique nous apparaissent avant tout être la visite guidée, la conférence, la démonstration spectaculaire. L'ensemble de ces trois propositions relève de la première posture du médiateur à savoir, de celui qui sait à celui qui demeure avant tout spectateur. Le discours est descendant ; le lieu est soit celui de l'exposition soit celui de la reconstitution du laboratoire. Si la visite prolonge l'univers des musées comme lieux avant tout patrimoniaux, les démonstrations spectaculaires rappellent fortement les grandes expériences scientifiques.

Les formats plus récents que sont l'atelier, le débat et le conseil/l'orientation portent la trace de l'entrée du visiteur dans l'espace de la médiation soit par la manipulation, la prise de parole ou encore l'écoute de la demande personnelle du visiteur. Ces formes plus récentes se distinguent des formes traditionnelles dans la mesure où elles laissent une plus grande place à la participation des visiteurs et répondent également au besoin d'individualisation des pratiques formulées par les visiteurs. Ainsi ces modes de médiation s'intègrent plus dans une logique de personnalisation de l'offre et la médiation présencielle renvoie à des problématiques sociales. Un exemple qui porte à l'extrême cette tendance consiste dans la médiation présencielle assurée au sein d'un espace comme celui de la Cité de la Santé (11): un personnel du corps médical reçoit de manière anonyme et gratuite un visiteur pour le conseiller, l'informer sur les démarches à suivre concernant sa santé ou celle d'un de ses proches. La médiation n'est plus dans ce cas précis au service d'une collection ou d'un travail muséographique qu'il s'agirait de magnifier ou de rendre accessible : elle est une offre autonome qui répond directement aux attentes des visiteurs.

De ces deux tendances émergent des formats hybrides comme celui du jeu, dans lequel le visiteur entre dans la démonstration par une participation ludique et où le médiateur n'intervient que pour conseiller, réguler et veiller à ce que les participants respectent les règles du jeu, ou encore dans celui de la facilitation où la visite ne s'opère plus face à un groupe mais bien dans un rapport individualisé entre le visiteur et le médiateur spécifiquement chargé de répondre aux besoins instantanés du visiteur et de l'orienter dans sa démarche de visite. 


\begin{tabular}{|c|c|c|c|c|}
\hline Place du médiateur & Place du visiteur & Relation médiateur/visiteurs & $\begin{array}{l}\text { Format de } \\
\text { la médiation }\end{array}$ & $\begin{array}{l}\text { Statut du } \\
\text { médiateur }\end{array}$ \\
\hline $\begin{array}{l}\text { Position frontale } \\
\text { du médiateur } \\
\text { (tenant du discours) }\end{array}$ & $\begin{array}{l}\text { Visiteur passif } \\
\text { (spectateur) }\end{array}$ & $\begin{array}{l}\text { Le médiateur fait face à son public, il est } \\
\text { le tenant du discours. Le mode de } \\
\text { communication est conférenciel : il ne laisse } \\
\text { pas de place à l'interaction, le visiteur reste } \\
\text { alors spectateur du dispositif, il est en position } \\
\text { de réception de la médiation. }\end{array}$ & $\begin{array}{l}\text { Conférence } \\
\text { Visite d'exposition }\end{array}$ & $\begin{array}{l}\text { Conférencier } \\
\text { Guide }\end{array}$ \\
\hline $\begin{array}{l}\text { Position médiane } \\
\text { du médiateur } \\
\text { (distribuer, réguler } \\
\text { le discours, } \\
\text { conseiller) }\end{array}$ & $\begin{array}{l}\text { Visiteur } \\
\text { à la fois actif } \\
\text { et passif } \\
\text { (acteur et } \\
\text { spectateur) }\end{array}$ & $\begin{array}{l}\text { Le médiateur est au centre : au milieu de son } \\
\text { public, il se tient à une distance raccourcie. } \\
\text { Sa position médiane et sa proximité avec le } \\
\text { public entraînent une participation du public } \\
\text { à l'élaboration du discours. Le médiateur est } \\
\text { présent pour réguler et distribuer la prise de } \\
\text { parole. Le visiteur participe plus ou moins } \\
\text { à la construction de la médiation. } \\
\text { Le déroulement prévoit plus ou moins } \\
\text { l'interaction avec et entre les visiteurs. }\end{array}$ & $\begin{array}{l}\text { Atelier débat, } \\
\text { démonstrations }\end{array}$ & $\begin{array}{l}\text { Animateur } \\
\text { Médiateur } \\
\text { Modérateur }\end{array}$ \\
\hline $\begin{array}{l}\text { Position de réception } \\
\text { du médiateur } \\
\text { (conseiller, aider, } \\
\text { orienter...) }\end{array}$ & $\begin{array}{l}\text { Visiteur actif } \\
\text { et en demande } \\
\text { (acteur) }\end{array}$ & $\begin{array}{l}\text { Le médiateur est quasi-absent de l'activité de } \\
\text { médiation. Il est présent pour conseiller et réguler } \\
\text { au besoin. Le visiteur participe à la médiation } \\
\text { par ses interactions : en ce sens, il peut être } \\
\text { considéré comme acteur de la construction de } \\
\text { la médiation. }\end{array}$ & $\begin{array}{l}\text { Entretiens } \\
\text { individualisés } \\
\text { de Cité de la Santé, } \\
\text { Cité des Métiers, } \\
\text { Médiathèque }\end{array}$ & \\
\hline
\end{tabular}

\section{La médiation présencielle : un monde en effervescence}

La médiation directe renvoie à une multitude d'activités qui sont également le fruit de mouvements politiques ou populaires dont l'histoire mériterait d'être écrite. La créativité dans ce domaine n'en est encore qu'à ses débuts : l'investissement des musées des sciences et des techniques dans le spectacle vivant, l'entrée de performance artistique dans une programmation évènementielle, le retour du conte... laissent entrevoir de nouvelles possibilités d'offres dans ces institutions.

La médiation présencielle rejoint également le monde de la médiation au sens strict en répondant à une demande sociale de plus en plus forte en ce qui concerne la maîtrise des outils numériques, la gestion des connaissances... Des musées de grande notoriété comme le Science museum à Londres qui a créé le Dana center, centre d'expérimentation sur les relations science et société, ont également investi dans ce type de programmation atypique et

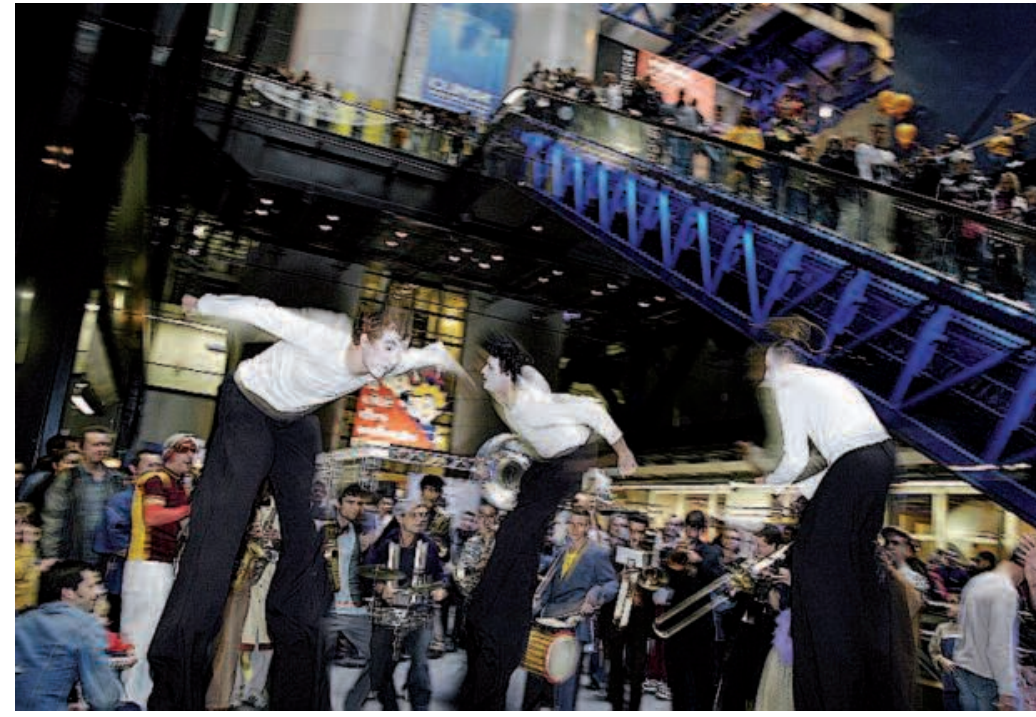

Animation de la Fête de la Science par une compagnie de théâtre de rue ○ CSI/B. Baudin, Le Bar Floréal 
laissent entrevoir une hybridation des formes et des formats de médiation (12).

Ces dispositifs qualitatifs qui reposent tous sur la rencontre d'un médiateur avec un ou des visiteurs sont un moyen de concrétiser la relation science et société en prenant en compte la diversité des demandes des publics d'une institution culturelle. Rappelons aux responsables de ces structures que même si ces propositions ont un coût pour une fréquentation qui peut apparaître modeste au regard d'autres dispositifs (13), ce média « chaud» qui cherche à impliquer le visiteur participe de l'image de l'institution et peut constituer une motivation supplémentaire - la rencontre humaine - à visiter un lieu de culture scientifique.

\section{Notes}

(1) Comme en témoigne la vivacité de cette revue.

(2) À l'exception du Palais de la Découverte qui, dans le monde des musées scientifiques, a repositionné son offre de médiation comme offre phare de son projet de rénovation, (cf. séminaire de muséologie à la CSI, le 20 janvier 2006).

(3) La professionnalisation des médiateurs, des animateurs, des guides conférenciers a suscité de nombreux débats qui ne sont toujours pas clos à l'heure actuelle. (cf. Les journées de réflexion sur les métiers de la médiation scientifique, $1^{\text {er }}$ et 2 septembre 2003, Palais de la Découverte). Une meilleure connaissance de la richesse et de la création de ces activités, ainsi que l'usage d'un vocabulaire commun faciliteraient une plus grande reconnaissance des compétences professionnelles mises en œuvre dans la conception et l'exploitation d'activités de médiation.

(4) Cet article est issu du rapport Belaën, F. Quelle signature pour la médiation humaine à la Cité ? Recensement, analyse, préconisations. Rapport CSI, 2006, 68 p. Pour connaître les autres conclusions de cette étude, le rapport ainsi que les études de publics sont disponibles à la médiathèque spécialisée en Histoire des Sciences de la CSI.

(5) Science museum à Londres, la Cosmocaixa à Barcelone, Exploratorium à San Francisco, le Palais de la Découverte et le musée des Arts et Métiers à Paris et la Cité de l'Espace à Toulouse.

(6) Précisons que nous avons finalement opté pour l'expression de «médiation présencielle ", celle-ci offrant l'avantage de rappeler la mise en jeu de savoirs, de savoir-faire mais également de savoir être (Caillet, 1994).

(7) Plusieurs types d'évaluations (études quantitatives, qualitatives, de type ethnographique) ont été menés entre 2002 et 2006. Les résultats sont consultables à la médiathèque de la CSI.

(8) L'Observatoire des Publics a été créé dès l'ouverture de la CSI par le département Évaluation et Prospective.

(9) Plus la forme est sophistiquée, plus le médiateur a recours à des artifices scéniques, mais rappelons toutefois que le principe de la médiation directe est avant tout celle de la rencontre du médiateur avec du public. (10) Rappelons que la sociabilité représente un moteur clef dans la visite d'un lieu culturel et qu'elle représente une activité importante au cours d'une visite dans un musée (Niquette, 2000).
(11) Voir les écrits d'Olivier Las Vergnas, responsable des départements Cité des Métiers et Cité de la Santé qui a innové en termes de médiation en mettant en œuvre le concept d'empowerment.

Voir http://mapage.noos.fr/siteolv/corps_zero.htm.

(12) Voir www.danacentre.org.uk/

(13) Les ateliers sont de loin moins efficaces que toute exposition grand public en terme de capacité d'accueil du public

\section{Bibliographie}

Belaën, F. 2006, Quelle signature pour la médiation humaine à la Cité ? Recensement, analyse, préconisations. Rapport CSI, 2006, 68 p.

Belaën, F. La médiation scientifique, à la recherche de méthodes d'analyse, in Journée d'étude Culture et Communication Approches des questions culturelles de l'information et de la communication, Collection UL3, 2004, pp. 103-109.

Belaën, F. Rapport d'évaluation sur des produits d'animation pendant les périodes de fortes fréquentations, Action Culturelle, CSI, 2003, 22 p.

Caillet, E. L'ambiguïté de la médiation culturelle : Entre savoir et présence, Publics \& Musées, n6, 1994, pp. 53-71.

Eidelmann, J. Politique de la science ou politique de l'esprit ? Genèse du Palais de la Découverte, La Science en scène, Paris : Presses de l'École normale supérieure et Palais de la Découverte, 1996.

Gaudillière, J.-P. La culture scientifique et technique, entre amateurs et profanes, Alliage, n59, 2006, pp. 3-9.

Niquette, M. Quand les visiteurs ne sont pas seuls : l'analyse sémiocognitive, in La muséologie des sciences et ses publics : Regards croisés sur la galerie de l'Évolution du Muséum national d'Histoire naturelle. Paris : Presses Universitaires de France (coll. Éducation et formation), 2000, pp. 181-198.

Raichvarg, D. et Jacques, J. Savants et ignorants. Paris : Seuil, 2003.

Raichvarg, D. Science et Spectacle, figure d'une rencontre. Nice : Z’Édition, 1993.

Schaer, R. L'invention des musées. Paris : Gallimard, 1993.

Schiele, B. L'invention simultanée du visiteur et de l'exposition, Publics $\&$ Musées, n², 1992, pp. 71-95.

Tievant, S. Analyse de la réception de produits d'animation, Rapport CSI, 1997.

Wacquet, F. Parler comme un livre. L'oralité et le savoir (XVI'-XXe siècles). Paris : Albin Michel, 2003. 